Grand Valley State University

ScholarWorks@GVSU

$3-5-2013$

\title{
Ultraviolet Photodissociation of OCS: Product Energy and Angular Distributions
}

George C. McBane

Grand Valley State University, mcbaneg@gvsu.edu

Johan A. Schmidt

University of Copenhagen, johanalbrechtschmidt@gmail.com

M. S. Johnson

University of Copenhagen

Reinhard Schinke

Max-Planck-Institut für Dynamik und Selbstorganisation, rschink@gwdg.de

Follow this and additional works at: https://scholarworks.gvsu.edu/chm_articles

Part of the Biological and Chemical Physics Commons

\section{ScholarWorks Citation}

McBane, George C.; Schmidt, Johan A.; Johnson, M. S.; and Schinke, Reinhard, "Ultraviolet Photodissociation of OCS: Product Energy and Angular Distributions" (2013). Peer Reviewed Articles. 25. https://scholarworks.gvsu.edu/chm_articles/25

This Article is brought to you for free and open access by the Chemistry Department at ScholarWorks@GVSU. It has been accepted for inclusion in Peer Reviewed Articles by an authorized administrator of ScholarWorks@GVSU. For more information, please contact scholarworks@gvsu.edu. 


\title{
Ultraviolet photodissociation of OCS: Product energy and angular distributions
}

\author{
G. C. McBane, ${ }^{1, a)}$ J. A. Schmidt, ${ }^{2, b)}$ M. S. Johnson, ${ }^{2}$ and R. Schinke ${ }^{3}$ \\ ${ }^{1}$ Department of Chemistry, Grand Valley State University, Allendale, Michigan 49401, USA \\ ${ }^{2}$ Department of Chemistry, University of Copenhagen, Universitetsparken 5, DK-2100 \\ Copenhagen $\emptyset$, Denmark \\ ${ }^{3}$ Max-Planck-Institut für Dynamik und Selbstorganisation (MPIDS), D-37077 Göttingen, Germany
}

(Received 31 December 2012; accepted 8 February 2013; published online 5 March 2013)

\begin{abstract}
The ultraviolet photodissociation of carbonyl sulfide (OCS) was studied using three-dimensional potential energy surfaces and both quantum mechanical dynamics calculations and classical trajectory calculations including surface hopping. The transition dipole moment functions used in an earlier study [J. A. Schmidt, M. S. Johnson, G. C. McBane, and R. Schinke, J. Chem. Phys. 137, 054313 (2012)] were improved with more extensive treatment of excited electronic states. The new functions indicate a much larger contribution from the $1^{1} A^{\prime \prime}$ state $\left({ }^{1} \Sigma^{-}\right.$in linear OCS) than was found in the previous work. The new transition dipole functions yield absorption spectra that agree with experimental data just as well as the earlier ones. The previously reported potential energy surfaces were also empirically modified in the region far from linearity. The resulting product state distributions $P_{v, j}$, angular anisotropy parameters $\beta(j)$, and carbon monoxide rotational alignment parameters $A_{0}^{(2)}(j)$ agree reasonably well with the experimental results, while those computed from the earlier transition dipole and potential energy functions do not. The higher- $j$ peak in the bimodal rotational distribution is shown to arise from nonadiabatic transitions from state $2{ }^{1} A^{\prime}$ to the OCS ground state late in the dissociation. (c) 2013 American Institute of Physics. [http://dx.doi.org/10.1063/1.4793275]
\end{abstract}

\section{INTRODUCTION}

The photodissociation of carbonyl sulfide (OCS) in the first absorption band $(\sim 222 \mathrm{~nm})$, a process of significant importance for atmospheric chemistry of the Earth, ${ }^{1,2}$ has been the subject of detailed experimental investigations. Brouard et $a l .{ }^{3}$ and Lipciuc et $a l .{ }^{4}$ have provided good recent reviews. The absorption spectrum has a broad Gaussian-like profile with pronounced vibrational structures. ${ }^{5,6}$ The carbon monoxide (CO) vibrational distribution, $P_{v}$, is very cold, not only at the low-energy onset of the spectrum but also for excitation energies near the maximum. ${ }^{7}$ In contrast, the measured rotational distributions, $P_{j}$, are hot; they are highly inverted and bimodal with a main bell-shaped peak at intermediate rotational states $j$ and a secondary peak at higher $j .{ }^{7,8}$ With increasing excitation energy, both peaks shift to higher $j$ and the intensity of the second one increases relative to the intensity of the main peak. The angular distribution of the products, characterized by the parameter $\beta$, has been measured for several wavelengths as function of $j .{ }^{3,4,7-12} \beta$ shows a strong variation with $j$ for all wavelengths. It has small positive values in the range of the main peak of the rotational distribution but increases steeply to almost the maximum possible value of 2 in the range of the second maximum of $P_{j}$. The interpretation of this peculiar $j$ dependence has varied, especially concerning the relative contributions of the excited ${ }^{1} A^{\prime}$ and ${ }^{1} A^{\prime \prime}$ states to the absorption cross section. ${ }^{3,4}$

\footnotetext{
${ }^{a)}$ Electronic mail: mcbaneg@ gvsu.edu.

b) Electronic mail: johanalbrechtschmidt@ gmail.com.
}

The wave packet dynamics calculations of Suzuki et al., ${ }^{8}$ using calculated potential energy surfaces (PES) and transition dipole moment (TDM) functions, provided a valuable overview of the electronic states involved and the general dissociation dynamics. However, neither the absorption cross section nor the product state distributions were satisfactorily reproduced. The angular distribution, a key quantity for understanding OCS photodissociation, was not considered at all.

Following our theoretical work on the UV photodissociation of $\mathrm{N}_{2} \mathrm{O},{ }^{13-17}$ which has the same number of 16 valence electrons, we recently began to study the complex photodissociation dynamics of OCS. ${ }^{18,19}$ We calculated global three-dimensional PESs for the four lowest singlet states (letters in parentheses denote abbreviations used below) $-1{ }^{1} A^{\prime}(\mathrm{X}), 2{ }^{1} A^{\prime}(\mathrm{A}), 1{ }^{1} A^{\prime \prime}(\mathrm{B})$, and $2{ }^{1} A^{\prime \prime}(\mathrm{C})$, — and the four lowest triplet states- $1^{3} A^{\prime}$ (a), $2^{3} A^{\prime}$ (d), $1{ }^{3} A^{\prime \prime}$ (b), and $2^{3} A^{\prime \prime}$ (c)—employing the multi-configuration reference internally contracted configuration interaction (MRCI) theory. ${ }^{20,21}$ The TDM functions with the ground state $\mathrm{X}$ were also calculated on three-dimensional grids. Details of the electronic structure calculations are described in Ref. 19.

The calculated absorption cross section ${ }^{19}$ agrees very well with the measured one. ${ }^{6}$ The overall shape of the spectrum, the peak position, the width, and the vibrational structures in the center and the low-energy and low-intensity part of the spectrum are all well described. The variations of the absorption spectrum with temperature and with $\mathrm{C}$ and $\mathrm{S}$ isotope substitution are also reproduced satisfactorily. ${ }^{2,22}$ The main results of the calculations can be summarized as follows: (a) Except for the onset where the intensity is extremely small, the absorption is dominated by excitation of the repulsive A 
state, while excitation of the repulsive B state is minor. (b) The vibrational structures in the center of the band are due to excitation of the triplet state $\mathrm{c}$, whose TDM is relatively large because of a near degeneracy with state A close to linearity. (c) The vibrational structures at the onset of the spectrum are due to the vibrational levels in the bent potential wells of both states A and B.

In this study we consider the $\mathrm{CO}$ product vibrational and rotational state distributions, and the angular distributions and their dependence on $j$. Calculations using the original PESs give rotational distributions that are only in qualitative agreement with the measured distributions; however, the peaks occur at $j$ values that are significantly too high. This deficiency points to failures of the angular dependences of the calculated potential energy surfaces $V_{\mathrm{A}}$ and $V_{\mathrm{B}}$. We apply a simple modification of the A- and B-state PESs that yields reasonable rotational distributions without changing the absorption cross section.

The $\beta(j)$ values computed using the modified potentials and TDM functions we reported before ${ }^{19}$ are in severe disagreement with the measured data. Instead of rapidly increasing with $j$ from small values to about the maximum possible value, as observed experimentally, they are almost constant, ranging from about 1.5 to 2 . The parameter $\beta$ depends sensitively on the relative contributions of the ${ }^{1} A^{\prime}$ states, for which $\beta$ can range from -1 to 2 , depending on the recoil dynamics and the direction of the transition dipole moment vector in the molecular plane, and the ${ }^{1} A^{\prime \prime}$ states, for which $\beta$ is equal to -1 for all $j$ independent of the dissociation path. Thus, the qualitative disagreement indicates that the calculated TDMs used in our previous investigations are faulty. New electronic structure calculations including higher excited electronic states than those of Ref. 19 indeed show that the ratio of TDMs, $\left|\boldsymbol{\mu}_{\mathrm{B}}\right| /\left|\boldsymbol{\mu}_{\mathrm{A}}\right|$, is significantly larger than determined before. With these new TDMs the calculated $\beta(j)$ is in reasonable agreement with experiment for several wavelengths; the total absorption cross section, however, remains almost unaffected by these changes.

In what follows, energies are given relative to the equilibrium of the $\mathrm{X}$ state, and the Jacobi coordinates $R$ (distance from $\mathrm{S}$ to the center of mass of $\mathrm{CO}$ ), $r$ (CO bond length), and $\gamma$ (angle between $\mathbf{R}$ and $\mathbf{r}$ ) are used.

\section{ELECTRONIC STRUCTURE CALCULATIONS}

Only the TDMs of the excited singlet states $2{ }^{1} A^{\prime}, 1^{1} A^{\prime \prime}$, and $2^{1} A^{\prime \prime}$, i.e., states $\mathrm{A}, \mathrm{B}$, and $\mathrm{C}$, with the ground state $\mathrm{X}$ have been recalculated. The TDMs of the triplet states a-d and the $a b$ initio PESs of all states involved are the same as in the previous studies. ${ }^{18,19}$ The nonadiabatic coupling matrix element (NACME) between excited state $\mathrm{A}$ and ground state $\mathrm{X}$ was also computed.

\section{A. New transition dipole moment functions for $A, B$, and C}

The new calculations for the TDMs of the singlet states $\mathrm{A}, \mathrm{B}$, and $\mathrm{C}$ with the ground state $\mathrm{X}$ were performed at the MRCI level of theory ${ }^{20,21}$ based on wave functions obtained by state-averaged full-valence complete active space self con- sistent field (CASSCF) calculations. ${ }^{23,24}$ The MRCI calculations used 12 active and 7 core orbitals. While in the previous TDM calculations the augmented correlation consistent polarized valence triple zeta (aug-cc-pVTZ) basis set of Dunning ${ }^{25}$ was employed, in the present study we used the aug-cc-pVTZ$\mathrm{d}$ basis, ${ }^{26}$ which includes an additional compact $d$-function on sulfur; however, the differences caused by this change in basis are quite small. The TDM for state A is a vector that lies in the molecular plane, whereas the TDMs for B and C are perpendicular to it. All electronic structure calculations were performed with the MOLPRO suite of programs. ${ }^{27}$

In the previous calculations of the TDMs ${ }^{18,19}$ the CASSCF orbitals were averaged over a "minimum" set of states: For $\boldsymbol{\mu}_{\mathrm{A}}$, for example, only the two states $1{ }^{1} A^{\prime}$ and $2{ }^{1} A^{\prime}$ were considered; likewise, the calculation of $\mu_{\mathrm{B}}$ and $\mu_{\mathrm{C}}$ included only the three states $1^{1} A^{\prime}, 1{ }^{1} A^{\prime \prime}$, and $2{ }^{1} A^{\prime \prime}$. This restriction provided a trustworthy description of the photoexcitation of $\mathrm{N}_{2} \mathrm{O} \cdot{ }^{13,17,28}$ Subsequent calculations, however, showed that for OCS higher states need to be included in the CASSCF averaging. The reason is probably intensity borrowing from the bright $4^{1} A^{\prime}$ state. This state is strongly mixed with $3{ }^{1} A^{\prime}$ in an avoided crossing in the Franck-Condon (FC) region. Convergence tests led to the conclusion that determining the orbitals by optimizing the energy average of the lowest four ${ }^{1} A^{\prime}$ and the lowest four ${ }^{1} A^{\prime \prime}$ states was sufficient. Including the fifth states of each symmetry did not alter the TDMs significantly. The new TDMs were computed on a fine three-dimensional grid in the Franck-Condon region and then interpolated with cubic splines for use in the dynamics calculations.

The extension of the states included in the CASSCF and MRCI calculations has two major effects. First, the TDM for state B is significantly enhanced while the TDM for state A is reduced. Second, in the FC region the vector $\mu_{\mathrm{A}}$ is considerably shifted away from the OCS axis, i.e., the ratio $\left|\boldsymbol{\mu}_{\mathrm{A}}^{y}\right| /\left|\boldsymbol{\mu}_{\mathrm{A}}^{z}\right|$ is increased. Both effects are clearly documented in Table I which shows the new and the old TDMs for A, B, and C as functions of $\gamma$ in the range important for excitation from the $\mathrm{X}$ state. They profoundly influence the angular distributions

TABLE I. Transition dipole moments (in atomic units) between the ground state $\mathrm{X}$ and states $\mathrm{A}, \mathrm{B}$, and $\mathrm{C}$ as functions of the bending angle $\gamma$ (in degrees) for $R=4.3 a_{0}$ and $r=2.2 a_{0}$.

\begin{tabular}{lcccc}
\hline \hline$\gamma$ & $\left|\boldsymbol{\mu}_{\mathrm{A}}^{y}\right| /\left|\boldsymbol{\mu}_{\mathrm{A}}^{z}\right|$ & $\left|\boldsymbol{\mu}_{\mathrm{A}}\right|$ & $\left|\boldsymbol{\mu}_{\mathrm{B}}\right|$ & $\left|\boldsymbol{\mu}_{\mathrm{C}}\right|$ \\
\hline 2.5 & $-2.08^{\mathrm{a}}$ & $2.67(-2)^{\mathrm{b}}$ & $2.75(-2)$ & $1.30(-2)$ \\
& -0.43 & $1.80(-2)$ & $6.43(-3)$ & $5.84(-3)$ \\
5 & -1.18 & $6.02(-2)$ & $5.37(-2)$ & $1.33(-2)$ \\
& -0.24 & $6.60(-2)$ & $1.63(-2)$ & $7.28(-3)$ \\
7.5 & -0.78 & $1.06(-1)$ & $7.29(-2)$ & $1.27(-2)$ \\
& -0.19 & $1.39(-1)$ & $2.61(-2)$ & $8.48(-3)$ \\
10 & -0.59 & $1.61(-1)$ & $8.67(-2)$ & $1.27(-2)$ \\
& -0.17 & $2.30(-1)$ & $3.56(-2)$ & $9.81(3)$ \\
12.5 & -0.48 & $2.18(-1)$ & $9.64(-2)$ & $1.32(-2)$ \\
& -0.17 & $3.24(-1)$ & $4.46(-2)$ & $1.13(-2)$ \\
15 & -0.41 & $2.74(-1)$ & $1.03(-1)$ & $1.04(-1)$ \\
& -0.16 & $4.10(-1)$ & $5.29(-2)$ & $1.29(-2)$ \\
\hline \hline
\end{tabular}

${ }^{a}$ First line: new calculations; second line: previous calculations. ${ }^{19}$

${ }^{\mathrm{b}}$ Numbers in parentheses indicate powers of 10 . 
as will be discussed in detail below. The TDM for $\mathrm{C}$ is also increased yielding a larger absorption cross section compared to the previous calculations.

It is interesting to note that the new TDMs, which are reasonably converged with respect to the number of states averaged over in the CASSCF calculations, agree satisfactorily with the results obtained with the equations-of-motion coupled-cluster method ${ }^{29}$ restricted to single and double excitations (EOM-CCSD) ${ }^{30}$ The EOM-CCSD approach usually gives good agreement with full CI calculations when the excited states are adequately described by promotion of a single electron from the ground state and the ground state itself is well described by a single-reference calculation. These conditions hold with reasonable accuracy for OCS near linearity. Therefore the agreement of the new MRCI and the EOMCCSD calculations confirms that the new TDMs are trustworthy and superior to the ones used in the earlier studies. ${ }^{18,19}$ For $\mathrm{N}_{2} \mathrm{O}$, on the other hand, the MRCI TDMs obtained by optimizing the energy average of only the minimum number of states in the CASSCF calculations already agree well with the TDMs obtained with the EOM-CCSD method. ${ }^{30}$ This indicates that for $\mathrm{N}_{2} \mathrm{O}$ the influence of the higher state wave functions on $\boldsymbol{\mu}_{\mathrm{A}}, \boldsymbol{\mu}_{\mathrm{B}}$, and $\boldsymbol{\mu}_{\mathrm{C}}$ is negligible.

The triplet state TDMs were not recalculated. Except for $\boldsymbol{\mu}_{\mathrm{c}}$, they are much smaller than the singlet state TDMs and affect the absorption cross section only in its far red wing. The c-state TDM is relatively large in the FC region due to near degeneracy with the A state at very small bending angles. ${ }^{19}$ Changes of the CASSCF wave functions in the MRCI calculations are not expected to significantly alter $\boldsymbol{\mu}_{\mathrm{c}}$.

\section{B. Nonadiabatic matrix elements}

The X-and the A-state PESs have an avoided crossing in the range of, depending on $R, \gamma=60^{\circ}$ to $90^{\circ}$ as illustrated in Fig. 1(a) where $V_{\mathrm{A}}-V_{\mathrm{X}}$ is shown for different $R$. Near
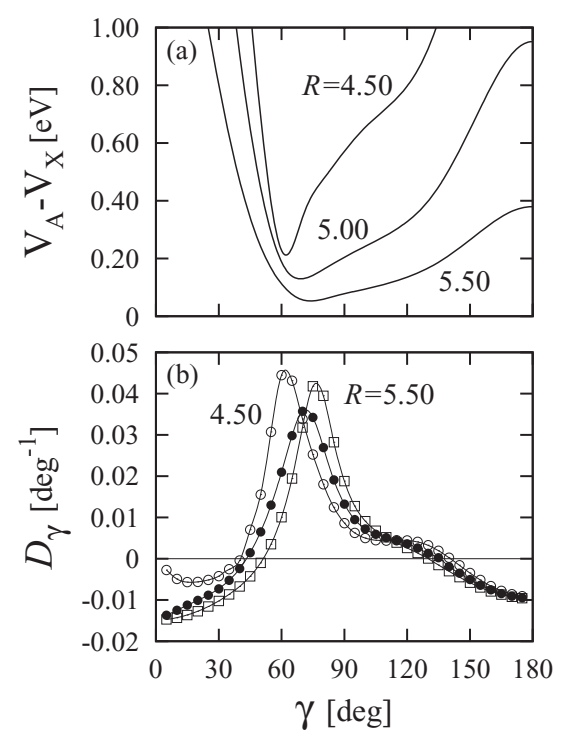

FIG. 1. (a) Energy separation $V_{\mathrm{A}}-V_{\mathrm{X}}$ vs. $\gamma$ for fixed $R$ as indicated and $r=2.2 a_{0}$. (b) NACME $D_{\gamma}$ vs. $\gamma$ for the same values of $R$ as in (a) and $r=2.2 a_{0}$. The symbols represent the calculated data points. this avoided crossing, transitions from the excited state A to the ground state $\mathrm{X}$, both having ${ }^{1} A^{\prime}$ symmetry, can happen while the molecule breaks apart. Suzuki et al. ${ }^{8}$ discussed the possibility of nonadiabatic $\mathrm{A} \rightarrow \mathrm{X}$ transitions and argued that the bimodality of the rotational state distributions is the result of such transitions. A similar, but much less pronounced effect was investigated by Schmidt et al. ${ }^{14}$ for $\mathrm{N}_{2} \mathrm{O}$.

In order to describe the $\mathrm{A} \rightarrow \mathrm{X}$ transitions in the dynamics calculations the NACMEs,

$$
D_{\xi}(R, r, \gamma)=\left\langle\Xi_{\mathrm{X}}|\partial / \partial \xi| \Xi_{\mathrm{A}}\right\rangle
$$

are required, where $\xi=R, r$, or $\gamma$ and $\Xi_{\mathrm{X}}$ and $\Xi_{\mathrm{A}}$ are the coordinate dependent adiabatic electronic wave functions for states A and X, respectively. ${ }^{31}$ We considered only the NACME with respect to the motion in $\gamma$, just as in our earlier work with $\mathrm{N}_{2} \mathrm{O} ;{ }^{14}$ justifications for neglecting the other matrix elements will be given below. Suzuki et al. ${ }^{8}$ applied the same approximation in their OCS calculations.

$D_{\gamma}$ was calculated using the MOLPRO suite of programs $^{27}$ at the CASSCF level and the aug-cc-pVTZ basis was employed. Figure 1(b) shows $D_{\gamma}$ versus $\gamma$ for several values of $R$ and $r=2.2 a_{0}$. The pronounced maxima correlate with the minima of the energy separation between $\mathrm{A}$ and $\mathrm{X}$ shown in (a). $D_{\gamma}$ was calculated on a two-dimensional grid with $0<\gamma<180^{\circ}$ and $4 a_{0} \leq R \leq 7 a_{0}$. Because the $\mathrm{CO}$ bond length is hardly changed during dissociation (see below), $r$ was fixed at $2.2 a_{0}$. Values between the calculated points were obtained by interpolation by cubic spline functions.

\section{ABSORPTION CROSS SECTION}

In order to determine the effects of the new TDMs on the absorption cross section we performed quantum mechanical wave packet calculations for excitation of the singlet states $\mathrm{A}, \mathrm{B}$, and $\mathrm{C}$, following the description given by Schmidt et al. ${ }^{19}$ The TDMs and, therefore, the cross sections for the four triplet states are the same as before. All electronic states were treated separately and possible couplings-nonadiabatic coupling, Renner-Teller coupling, and spin-orbit couplingwere neglected. In all calculations the total angular momentum was set to zero. Lifetime and rotational broadening of the bound states $\mathrm{C}$, c, and d were empirically accounted for by convolving the corresponding cross sections with a Gaussian with full width at half maximum of $125 \mathrm{~cm}^{-1}$. In order to compute thermal cross sections, absorption cross sections for the lowest five vibrational states of $\mathrm{X}$ were determined. The numerical parameters such as grid size and propagation time were the same as in Ref. 19.

Figure 2(b) shows the $170 \mathrm{~K}$ absorption cross sections for states $\mathrm{A}, \mathrm{B}$, and $\mathrm{C}$ ( $\sigma_{\mathrm{A}}$ etc.) vs. photon energy $E_{\mathrm{ph}}$ obtained with the new TDMs; the cross section for the triplet state $\mathrm{c}$ is also shown. As in Ref. 19, for comparison with experiment the calculated cross section for state A was shifted by $200 \mathrm{~cm}^{-1}$ to higher energies. The cross sections for the other electronic states were not shifted. The excitation energies from the ground state may not be of the same accuracy for the different states. 

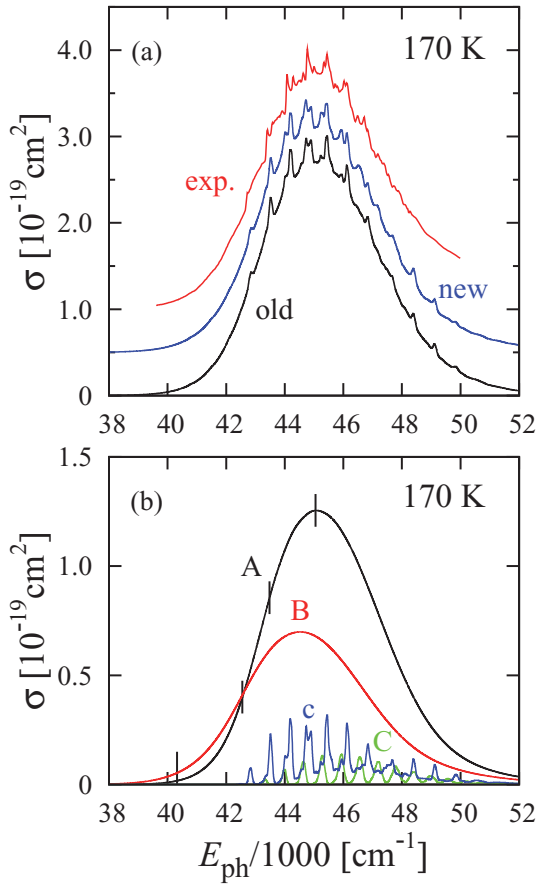

FIG. 2. (a) Comparison of the new absorption cross section (multiplied by 1.3), the old cross section from Ref. 19 (multiplied by 1.4), and the experimental cross section ${ }^{6}$ for $T=170 \mathrm{~K}$. For clarity of the presentation the new cross section and the experimental one are shifted upward by 0.5 and 1.0 $\times 10^{-19}$, respectively. (b) Absorption cross sections for states A (black), B (red), and C (green) obtained with the new TDMs for $T=170 \mathrm{~K}$; the cross section for the triplet state $\mathrm{c}$ (blue) is also shown. The short vertical lines indicate the wavelengths at which rotational state and angular distributions have been measured: 248, 235, 230, and $222 \mathrm{~nm}$. See the text for more details.

Dissociation via states $\mathrm{A}$ and $\mathrm{B}$ is fast and direct and therefore the cross sections are structureless. States $\mathrm{C}$ and $\mathrm{c}$ are bound and $\sigma_{\mathrm{C}}$ and $\sigma_{\mathrm{c}}$ both exhibit vibrational structures, ${ }^{31}$ which reflect OC-S stretch coupled to the bending degree of freedom. ${ }^{18}$ For comparison, the structures of the $\mathrm{N}_{2} \mathrm{O}$ UV absorption spectrum reflect strongly mixed bending and $\mathrm{N}_{2}$ motion in the dissociative A state. ${ }^{13,32}$

The cross sections of Fig. 2(b) have to be compared to those in Fig. 11 of Ref. 19. The main difference relative to the previous calculations is the dramatic increase of $\sigma_{\mathrm{B}}$ relative to $\sigma_{\mathrm{A}}$. While $\sigma_{\mathrm{A}}$ is still the larger cross section at the center of the band and at short wavelengths, $\sigma_{\mathrm{B}}$ dominates at the onset of the spectrum. The $\mathrm{C}$-state cross section is also increased in comparison to the old calculations and is now of the same order of magnitude as $\sigma_{\mathrm{c}}$. The vibrational structures arising from excitation of $\mathrm{C}$ and $\mathrm{c}$ are slightly shifted relative to each other.

The new total absorption cross section, summed over all the individual cross sections of Fig. 2(b), is compared to the old one and to the experimental cross section ${ }^{6}$ in Fig. 2(a). The new cross section was scaled by 1.3 , while the old one was multiplied by a slightly larger factor, 1.4. The overall underestimation by the calculations is not unusual ${ }^{13,33}$ and most likely reflects deficiencies of the TDMs. The differences between the old and the new absorption spectra are tiny. Because the structures from states $\mathrm{c}$ and $\mathrm{C}$ are slightly shifted relative to each other, the vibrational structures in the total spectrum are marginally more pronounced. Because the energies and amplitudes of the diffuse structures depend directly on subtleties of (at least) two PESs of different accuracies, we cannot expect to reproduce them perfectly. Altogether, the new total cross section seems to be in slightly better agreement with the measured cross section.

\section{PRODUCT STATE DISTRIBUTIONS}

Vibrational, $P_{v}$, and rotational, $P_{j}$, state distributions for particular wavelengths were determined from wave packet calculations as described in Ref. 14 for the dissociation of $\mathrm{N}_{2} \mathrm{O}$. Only the dissociative states A and B were considered. The cross sections for excitation of the bound states $\mathrm{C}$ and $\mathrm{c}$ (or the other triplet states $\mathrm{a}, \mathrm{b}$, and d) are much smaller and therefore their contributions to the product distributions can reasonably be neglected. The uncertainties resulting from the uncertainties of the A- and B-state PESs are larger than the contributions of these other states. Moreover, inclusion of the bound states would require wave packet calculations on several coupled PESs.

In addition to the quantum mechanical calculations the product state distributions were also calculated by means of classical trajectories as outlined in Ref. 14. Classical calculations were performed both without the nonadiabatic coupling to the $\mathrm{X}$ state and including it by means of the trajectory surface hopping (TSH) method. ${ }^{34,35}$ In the TSH method the coupling between states A and X was taken as $\dot{\gamma} D_{\gamma}$, where $D_{\gamma}$ is the NACME defined in Eq. (1). In Ref. 17 it was shown for $\mathrm{N}_{2} \mathrm{O}$ that the coupling term with respect to motion in $R$ is much smaller and the same is assumed for OCS; because of the considerably smaller excess energy and the higher reduced mass the velocity $\dot{R}$ is smaller for OCS dissociation. The $\mathrm{CO}$ vibration remains almost unexcited along the trajectory (see below) and therefore the coupling with respect to $r$ also can be neglected. In all dynamics calculations the total angular momentum $J$ was set to zero and the initial state was the ground vibrational state $(0,0,0)$ in $\mathrm{X}$.

\section{A. Vibrational state distributions}

The $\mathrm{CO}$ equilibrium bond distance in the $\mathrm{X}$ state as well as in the two excited states $\mathrm{A}$ and $\mathrm{B}$ is about $2.20 a_{0}$ and remains almost the same along the entire dissociation path (Figs. 4 and 5 in Ref. 19). Consequently, excitation of motion along $r$ is very weak and the final $\mathrm{CO}$ vibrational distributions are extremely cold. The quantum mechanical probabilities for excitation of $v=1$ at the wavelengths 248, 235, 230, and $222 \mathrm{~nm}$ are $0.01 / 0.03,0.02 / 0.005,0.04 / 0.02$, and $0.11 / 0.06$ where the first and second numbers refer to states A and B, respectively. The probabilities for higher states are basically zero. The classical results from the single-state calculations are similar. The very weak vibrational excitation is in accord with experiment: ${ }^{7}$ the measured probability for $v=1$ for $222 \mathrm{~nm}$ is merely 0.02 . The results for OCS are quite different from those for $\mathrm{N}_{2} \mathrm{O}$. The $\mathrm{N}_{2} \mathrm{O}$ vibrational distributions are also cold at long wavelengths. With decreasing wavelength, however, $P_{v}$ becomes inverted and the peak gradually shifts to higher $v .{ }^{14}$ The shape of the A-state PES, which includes 
a valley leading toward the $\mathrm{N}+\mathrm{NO}$ product channel that is absent in OCS, readily explains the strong wavelength dependence of $P_{v}$ for $\mathrm{N}_{2} \mathrm{O}$.

\section{B. Rotational state distributions}

\section{Single state calculations with the original PESs}

Because of the very weak vibrational excitation we will only consider rotational state distributions for $\mathrm{CO}(v=0)$ in what follows. The quantum mechanical rotational state distributions, for both A and B states, are highly inverted with a Gaussian-like shape; they are qualitatively similar to those observed in the photodissociation of $\mathrm{N}_{2} \mathrm{O} .{ }^{14}$ Examples are shown in Fig. 3(a) for excitation at $235 \mathrm{~nm}$. Because the Aand B-state PESs are quite similar, the rotational distributions are also similar. With decreasing wavelength the distributions shift to higher $j$ and become narrower.

The $P_{j}$ obtained from the trajectory calculations are qualitatively similar but they are systematically shifted to higher $j$; the difference is $\Delta j=3-4$ for $248 \mathrm{~nm}$ and $2-3$ for $223 \mathrm{~nm}$, corresponding to energies of approximately $0.08 \mathrm{eV}$. In view of the almost perfect decoupling of the $\mathrm{CO}$ vibrational degree of freedom from translation and rotation, manifested by the weak vibrational excitation, we believe that unphysical distribution of the zero-point energy of $\mathrm{CO}(0.134 \mathrm{eV})$ into rotation leads to the shift in the classical rotational distributions. A reduction of the excitation energy by $0.1 \mathrm{eV}$, i.e., $75 \%$ of the zero-point energy, in the classical calculations yields reasonable agreement between the quantal and the classical distributions for the four wavelengths considered below. The value of $0.1 \mathrm{eV}$ is an ad hoc correction term; it is not the result
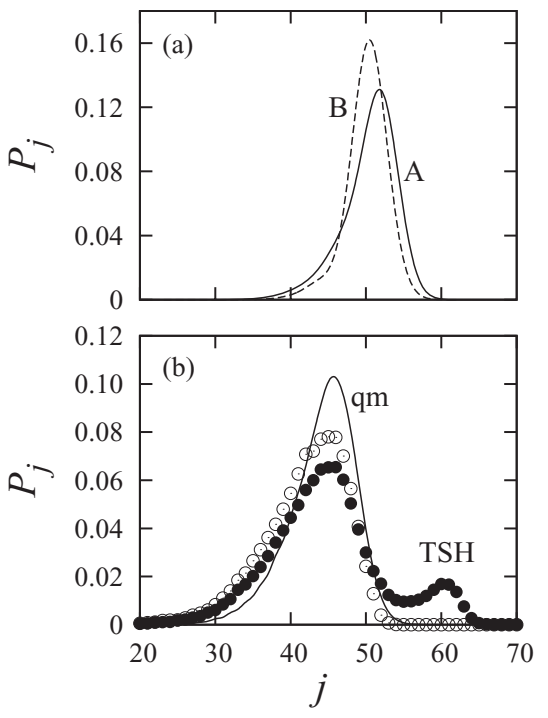

FIG. 3. (a) Quantum mechanical rotational state distributions for excitation with $\lambda=235 \mathrm{~nm}$ light. Comparison of $P_{j}$ for dissociation in the A state (not including the coupling to $\mathrm{X}$ ) and the $\mathrm{B}$ state. In both cases the original PESs were employed. The vibrational state is $v=0$. (b) Comparison of the quantum mechanical (qm, solid line) and the classical (open circles) rotational distributions for state A using the modified PES $V_{\text {mod }}$. Also shown is the result of the TSH calculation including the nonadiabatic $\mathrm{A} \rightarrow \mathrm{X}$ calculation (filled circles). $\lambda=235$ and $v=0$. The classical calculation used a total energy $0.1 \mathrm{eV}$ lower than the quantum one (see text.)
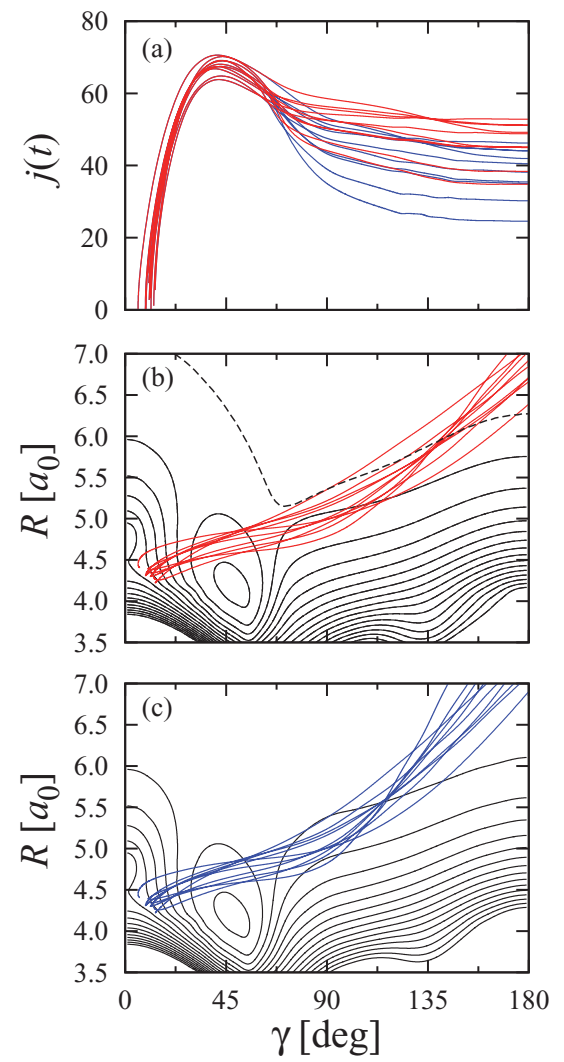

FIG. 4. (a) The classical rotational quantum number $j(t)$ as function of $\gamma$ for ten random trajectories for $\lambda=235 \mathrm{~nm}$ and dissociation in the A state. Red curves show results on the ab initio potential; blue curves show results on the potential after the empirical modification at $\gamma>60^{\circ}$. The initial conditions of the trajectories are identical for the two sets. (b) Contour plot of the ab initio A-state PES for fixed $r=2.2 a_{0}$. The same ten trajectories shown in (a) are superimposed. The dashed line indicates the avoided crossing between the $\mathrm{X}$ and the A PESs. (c) The same as in (b) for the empirically modified A-state PES.

of an extensive analysis. In all the classical calculations presented below the true excitation energy was lowered by this amount. The total energies used in the classical calculations were 5.144, 5.421, 5.536, 5.705, and $5.730 \mathrm{eV}$ for 248, 235, 230,223 , and $222 \mathrm{~nm}$ respectively.

In order to understand the dynamics leading to the bellshaped, highly inverted distributions it is helpful to analyze individual trajectories. In Fig. 4(a) we show, for excitation of state A with $235 \mathrm{~nm}$ light, the "classical rotational quantum number" $j(t)$ along ten typical trajectories. Figure 4(b) shows the corresponding trajectories in the $(R, \gamma)$ plane overlaid on the PES contours. $j(t)$ rises very quickly from the FC region to the bottom of the potential well near $45^{\circ}$. After passing the well the torque $\partial V / \partial \gamma$, which governs $j(t),{ }^{31}$ changes sign and $j(t)$ is significantly decreased with the result that the final value is much lower than the maximum value. The maximum value increases only slightly from $248 \mathrm{~nm}$ to $222 \mathrm{~nm}$. The deceleration, however, depends quite significantly on the dissociation path and therefore on the starting points of the trajectories and thus on the energy with which OCS is excited. It is strongest at the longest wavelength, but becomes smaller with decreasing $\lambda$. At $222 \mathrm{~nm}$, for example, the trajectories cross the well at a larger bond length $R$, hit the repulsive wall around $90^{\circ}$ also at larger $R$, and as a consequence they are 
less strongly deflected. The trajectories for $\mathrm{N}_{2} \mathrm{O}$ show a similar behavior, but the deceleration in the second part of the dissociation is much smaller. ${ }^{14}$

The agreement of the calculations using the original A and B PESs with the measured $P_{j}$ is poor: the calculated distributions peak at rotational states which are significantly too high. For example, the main peak of the measured distribution ${ }^{8}$ for $248 \mathrm{~nm}$ occurs at $j=33$ while the quantum mechanical distributions for A and B peak at 38 and 39, respectively. The corresponding numbers for $223 \mathrm{~nm}$ are 54 and 62/61. This disagreement clearly indicates that the PESs have some deficiencies. In the following we describe ad hoc modifications of the A- and B-state PESs which lead to reasonable agreement with the experimental $P_{j}$.

\section{Single state calculations with modified PESs}

As one can imagine from the trajectories in Fig. 4(b), the final $j$ depends sensitively on the path that a trajectory follows from the FC region through the well to the repelling potential branch around $90^{\circ}$ and beyond. Changing the PES near the FC point alters the route of dissociation and thus the final $j$ distribution, as we checked in test calculations. However, such modifications also change the absorption spectrum, destroying the good agreement the current PESs give with the experimental spectrum. Instead we chose to modify the repulsive part of the potential beyond the potential minimum. This modification did not alter the computed absorption spectrum.

The modification of both PESs consists of adding an exponential repulsion term along $R$ in the region from $\gamma=60^{\circ}$ to $180^{\circ}$. Between $\gamma=60^{\circ}$ and $95^{\circ}$ the exponential is smoothly switched on from zero to unity. The modified potential is defined by

$$
V_{\text {mod }}(R, r, \gamma)=V(R, r, \gamma)+\chi f(\gamma) \mathrm{e}^{a(R-z(\gamma))} .
$$

$z(\gamma)=4.4 a_{0}$ for $\gamma=60^{\circ}$ and $5.5 a_{0}$ for $\gamma=180^{\circ}$; between these points $z(\gamma)$ is interpolated by a linear function. The switching function is given by

$$
f(\gamma)=\sin ^{2}(\alpha \gamma-\beta)
$$

with the parameters $\alpha$ and $\beta$ chosen so that $f\left(60^{\circ}\right)=0$ and $f\left(95^{\circ}\right)=1$. The prefactor $\chi$ in Eq. (2) was adjusted by comparison with the experimental rotational distribution at $235 \mathrm{~nm}$ and finally was set to $0.2 \mathrm{eV}$. The steepness parameter $a$, defined by $-\ln (2) / \delta$, was found to have only a weak influence on the results; throughout the calculations $\delta$ was set to 0.75 . The same parameters were used for both $V_{\mathrm{A}}$ and $V_{\mathrm{B}}$.

Figure 4(c) shows contour plots of the modified A PES, for comparison with the original PES shown in Fig. 4(b). The region below $60^{\circ}$ is unchanged. The influence of the modification is clearly demonstrated by the trajectories overlayed to the potential contours: they are more strongly repelled at large $\gamma$ leading to a larger deceleration of $j(t)$ and therefore to smaller final $j(t)$ values [Fig. 4(a)]. The consequences of this additional rotational deceleration are apparent in a comparison of the quantum mechanical rotational distributions $P_{j}$ in the upper and lower panels of Fig. 3. The distributions for state $\mathrm{A}$ and $\lambda=235 \mathrm{~nm}$ obtained with the modified PES are substantially shifted to lower $j$ and agree much better with the experimental distribution for this wavelength, which peaks at $j \approx 44$ (see below). The shift to lower $j$ values is accompanied by some broadening of the distributions.

\section{Trajectory surface hopping calculations}

All rotational distributions, calculated on the A or B states using either the original or the modified potentials, are bell-shaped while the measured distributions are clearly bimodal. ${ }^{7,8}$ Suzuki et al. ${ }^{8}$ proposed that the second maximum at higher rotational states might be due to nonadiabatic transitions from state $\mathrm{A}$ to the ground state $\mathrm{X}$ at intermediate OC-S separations. (Nonadiabatic transitions from B to $\mathrm{X}$ do not take place because these states have different spatial symmetries.) TSH calculations including A-X nonadiabatic coupling unambiguously confirm their suggestion. An example is given in Fig. 3(b) showing $P_{j}$ for $235 \mathrm{~nm}$. The second peak at $j=60$ stems from trajectories which "hop" at least once from A to $\mathrm{X}$ during dissociation. If the hop occurs before the trajectory hits the repulsive wall of the A-state PES around $70^{\circ}$, the rotation is much less decelerated in the second part of the bond breaking; the X PES is more isotropic, i.e., $\partial V_{X} / \partial \gamma$ is small for larger angles (Fig. 2 of Ref. 19), and hence $j(t)$ does not change much on $V_{\mathrm{X}}$. Such trajectories create the second maximum at high $j$.

The seam of avoided crossing is indicated in Fig. 4(b). Our empirical modification of the A PES moves the X/A crossing seam to larger $R$ and therefore the NACME function and the X/A potentials are not fully consistent. However, tests at $235 \mathrm{~nm}$ indicated that this inconsistency has only a small effect on the rotational distributions. The position of the second peak, which definitely originates from the $\mathrm{A} \rightarrow \mathrm{X}$ transition, is not changed.

A similar mechanism leads to a small shoulder at the high- $j$ end of the main maximum of $P_{j}$ in the photodissociation of $\mathrm{N}_{2} \mathrm{O} .{ }^{14}$ However, the effect is much weaker than for OCS and somewhat different; while for OCS the rotation of $\mathrm{CO}$ is less decelerated on $V_{\mathrm{X}}$, the rotation of $\mathrm{N}_{2}$ is slightly more accelerated after the transition to $\mathrm{X}$.

\section{Comparison with measured distributions}

For comparison with the measured distributions we calculated the distributions for state $\mathrm{A}$, including transitions to $\mathrm{X}$, and state $\mathrm{B}$ for initial vibrational state $(0,0,0)$ and using the modified PESs. The results, including the contributions from excitation to individual states $\mathrm{A}$ and $\mathrm{B}$, are shown in the lower panels of Fig. 5 for wavelengths $248 \mathrm{~nm}, 235 \mathrm{~nm}, 230 \mathrm{~nm}$, and $223 \mathrm{~nm}$. The sum of the two individual distributions are compared with the experimental $P_{j}$. The experimental populations have been scaled so that the sum of the measured populations is unity.

The modification of the PESs at large angles described earlier was chosen to give a rotational distribution that agreed with experiment for the intermediate wavelength of $235 \mathrm{~nm}$. The adjustment also produces good agreement with the experimental distribution at $230 \mathrm{~nm}$. At $248 \mathrm{~nm}$ the main peak appears at smaller rotational states than that of the experimental distribution, while for $223 \mathrm{~nm}$ the main peak occurs 

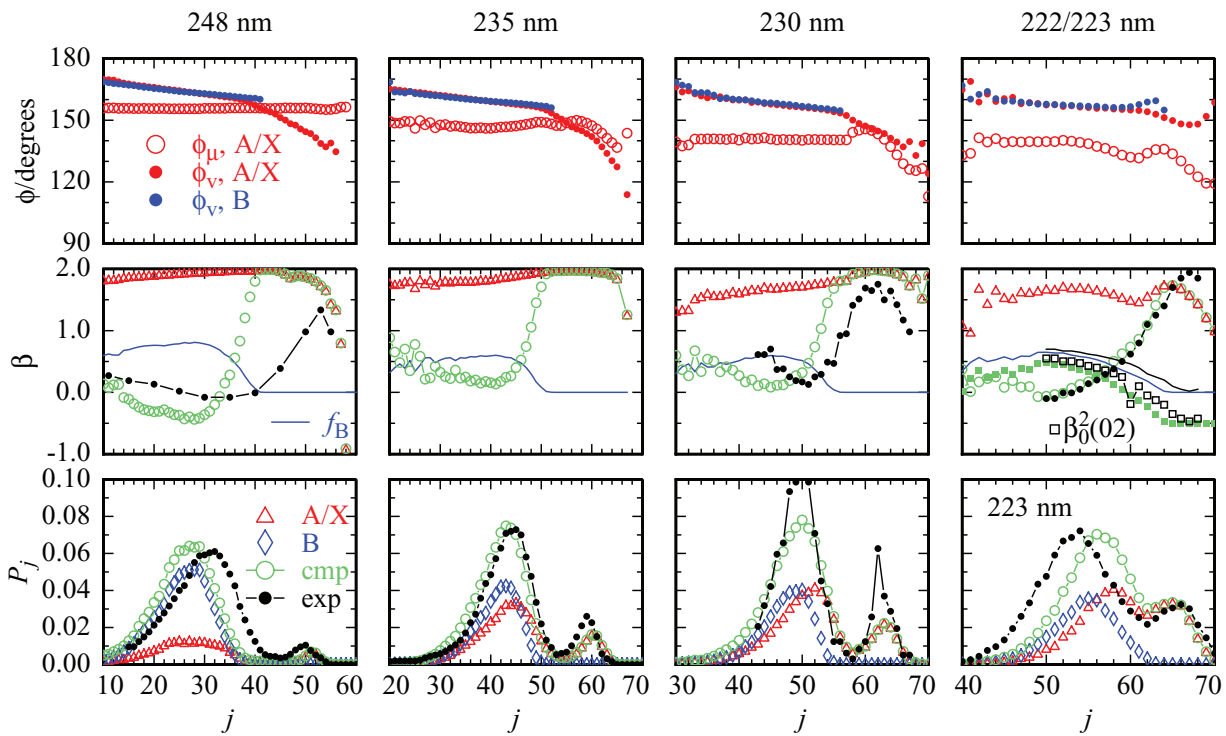

FIG. 5. Lower panels: CO rotational state distributions. Distributions computed for excitation to the A and B states separately, the composite distribution weighted according to the ratio of absorption cross sections, and experimental results are shown. Trajectories following excitation to A included the possibility of surface hops to the X state. The experimental results for 248, 235, and $223 \mathrm{~nm}$ come from Suzuki et al. ${ }^{8}$ those for $230 \mathrm{~nm}$ come from van den Brom et al. ${ }^{36}$ Center panels: Anisotropy parameters $\beta(j)$ computed from the same trajectories. Values computed from excitation to the A state, and composite values reflecting excitation to both $\mathrm{A}$ and $\mathrm{B}$, are shown along with experimental results. ( $\beta$ for pure $\mathrm{B}$ state excitation is always -1$)$. The solid blue curve shows the computed fractional contribution of B state excitation at each $j$. Experimental results are from Brouard et al. ${ }^{3}$ at $248 \mathrm{~nm}$, van den Brom et al. ${ }^{36}$ at $230 \mathrm{~nm}$, and Sivakumar et al. ${ }^{7}$ at $222 \mathrm{~nm}$. The solid black line at $222 \mathrm{~nm}$ shows the experimental estimate ${ }^{7}$ of the fractional contribution of an $A^{\prime \prime}$ state to the absorption. At $222 \mathrm{~nm}$ the $\mathrm{CO}$ angular momentum alignment bipolar moment $\beta_{0}^{2}(02)$ of Sivakumar et al. ${ }^{7}$ (open black squares), and the corresponding computed moment (filled green squares), are also shown. Upper panels: the orientation angles $\phi_{\mu}$ of the transition dipole moment and $\phi_{v}$ of the final S atom velocity, averaged over all trajectories in each rotational bin. The "single-trajectory $\beta$ " for A-state absorption is $2 P_{2}\left(\phi_{v}-\phi_{\mu}\right)$, while that for B-state absorption is -1 .

at slightly too high a $j$. Altogether, the agreement for all four wavelengths is reasonable; in particular the bimodal structure is well reproduced. More sophisticated modifications might give better quantitative agreement; however, they most likely would include more adjustable parameters, and it is not clear that they would aid in physical interpretation. Rotational distributions computed from the unmodified PESs are shifted to higher $j$ for all wavelengths, and in fact for $\lambda \leq 230 \mathrm{~nm}$ the secondary peak arising from nonadiabatic transitions is buried under the main peak and no longer discernable.

\section{PRODUCT ANGULAR DISTRIBUTIONS}

\section{A. Physical model}

Our classical model treats the nuclear dynamics occurring on one surface $(\mathrm{A}, \mathrm{B}$, or $\mathrm{X})$ at a time, with total angular momentum zero, and includes the possibility of nonadiabatic transitions between $\mathrm{A}$ and $\mathrm{X}$ as described earlier. We neglect the possibility of coherent simultaneous excitation of the A and B surfaces with subsequent interference between trajectories on the two surfaces. ${ }^{4,37-40}$ In addition, the $J=0$ assumption implies that the plane containing the three atoms is fixed in space during the dissociation. This model is not suited for predicting the distribution of electronic angular momentum vectors of the $S\left({ }^{1} D\right)$ product, ${ }^{3,4,8,12,39,41}$ and we do not address those measurements here.

We computed the anisotropy parameter $\beta$ following excitation to the A state using the procedure we previously described for $\mathrm{N}_{2} \mathrm{O}$ photodissociation. ${ }^{17}$ This parameter describes the distribution of product velocity vectors about the polarization vector of the dissociating light according to ${ }^{42}$
$I(\theta) \propto 1+\beta P_{2}(\cos \theta)$, where $P_{2}(x)$ is a Legendre polynomial and $\theta$ is the angle between the product velocity and electric field vectors. The transition dipole moment for this $A^{\prime} \leftarrow A^{\prime}$ transition must lie in the triatomic plane. The $y$ and $z$ components of the transition dipole moment in the principal axis frame of the electronic structure calculations were interpolated with three-dimensional cubic splines for each initial geometry. They were then transformed to the laboratory axis system used for the trajectory calculations, which had its $Z$ axis along the initial $R$ vector pointing from $\mathrm{S}$ to the center of mass of $\mathrm{CO}$. The components of the final lab-frame $\mathrm{S}$ atom velocity were determined from the terminal position and momentum quantities for each trajectory according to formulae given in the Appendix of Ref. 43. The angle $\theta_{\mathrm{m}}$ between the transition dipole direction and the final $\mathrm{S}$ atom velocity was determined for each trajectory, and the value $\beta$ was obtained for all trajectories finishing in a given $v, j$ bin from $\beta=2\left\langle P_{2}\left(\cos \theta_{\mathrm{m}}\right)\right\rangle$, where the angle brackets indicate a weighted average over trajectories. ${ }^{17,42}$ This procedure was applied to all trajectories beginning on the A state whether they made nonadiabatic transitions to $\mathrm{X}$ or not.

For absorption to the $\mathrm{B}$ state, no special calculations for $\beta$ are required, because for an $A^{\prime \prime} \leftarrow A^{\prime}$ transition the transition dipole vector must lie perpendicular to the triatomic plane, and the $J=0$ assumption implies that the final product velocity must lie in the plane. $\theta_{\mathrm{m}}$ must therefore be $\pi / 2$ for all dissociations resulting from $\mathrm{B}$ state absorption, and the corresponding $\beta=-1$.

Similarly, the alignment parameter $A_{0}^{(2)}$, which describes the distribution of $\mathrm{CO}$ rotational angular momentum vectors about the electric field vector, takes simple limiting values for 
excitation to both A and B states. ${ }^{7,44}$ For A state absorption, the transition dipole $\mu_{\mathrm{A}}$ lies in the triatomic plane while the rotational angular momentum vector $\boldsymbol{j}$ of $\mathrm{CO}$ is necessarily perpendicular to it. $A_{0}^{(2)}$ therefore takes its limiting negative value for one-photon photodissociation, $-2 / 5$. For the $\mathrm{B}$ state, $\mu_{\mathrm{B}}$ and $\boldsymbol{j}$ are necessarily parallel and $A_{0}^{(2)}=4 / 5$. To facilitate direct comparison with the experimental results of Sivakumar et al. ${ }^{7}$ we report alignment parameters in the form of the equivalent bipolar moment ${ }^{45} \beta_{0}^{2}(02)=\frac{5}{4} A_{0}^{(2)}$.

To construct composite values of the anisotropy or alignment parameters for comparison to experiment, we used weighted averages of the values arising from excitations to A or B. The weights represented the fractional contributions of absorption to the two electronic states to a particular final state of $\mathrm{CO}$. If $P_{\mathrm{A}}(j)$ and $P_{\mathrm{B}}(j)$ are separately normalized rotational distributions arising from excitation to the $\mathrm{A}$ and $\mathrm{B}$ states respectively, then the fractional contribution of $\mathrm{B}$ state excitation to final state $j$ is $f_{\mathrm{B}}=\sigma_{\mathrm{B}} P_{\mathrm{B}}(j) /\left(\sigma_{\mathrm{A}} P_{\mathrm{A}}(j)\right.$ $\left.+\sigma_{\mathrm{B}} P_{\mathrm{B}}(j)\right)$. The composite $\beta$ for that rotational state is then $\beta=\left(1-f_{\mathrm{B}}\right) \beta_{\mathrm{A}}+f_{\mathrm{B}} \beta_{\mathrm{B}}$. The same procedure was used to construct the composite alignment parameter $\beta_{0}^{2}(02)(j)$. The expression for $f_{\mathrm{B}}$ was rewritten in terms of cross section ratios $\sigma_{\mathrm{A}} / \sigma_{\mathrm{B}}$; these ratios were obtained from our computed absorption cross sections and were $0.390,1.146,1.506,1.784$, and 1.8106 at 248, 235, 230, 223, and $222 \mathrm{~nm}$, respectively.

\section{B. Anisotropy parameter}

The center panels of Fig. 5 show the anisotropy parameters $\beta$ computed for excitation to the A state, the composite ones reflecting excitation to both $\mathrm{A}$ and $\mathrm{B}$, the fractional contribution of B state absorption to each rotational level, and experimental measurements from several laboratories. ${ }^{3,7,36}$ At all wavelengths excitation to the A state produces $\beta$ near its limiting value of 2 across most of the rotational distribution, with a steep drop near the upper $j$ limit. The rotational distribution for the B state peaks at slightly lower $j$ than the corresponding one from dissociation on the A state, and its fractional contribution is therefore substantial for lower- $j$ trajectories even when its absorption cross section is smaller than that of the A state. The corresponding weighted average gives $\beta$ values that are low, on the order of zero, at low $j$ and rise to near-limiting values in the upper part of the rotational distribution. Beyond the central minimum in the rotational distribution, the $\mathrm{B}$ state makes no contribution and the anisotropy parameters reflect purely the $\mathrm{A} / \mathrm{X}$ dissociations.

The composite $\beta(j)$ values share the qualitative features of the experimental curves: $\beta$ is near zero in the low- $j$ tail, increases rapidly to near 2 between the first and the second maximum in $P_{j}$, and falls again near the upper $j$ limit. At $248 \mathrm{~nm}$ the quantitative agreement is not particularly good, possibly because our B state rotational distribution peaks at too low a $j$ and therefore the fractional contribution of the $\mathrm{B}$ state is underestimated between $j=30$ and 45 . Nonetheless the shape is correct, in contrast to an A-only model which completely fails to capture most of the variations in $\beta$ with $j$. At $230 \mathrm{~nm}$ the agreement is quite good except that the structure in the computed $\beta(j)$ is slightly too broad, reflecting the same disagreement in the rotational state distributions. The remarkably close agreement at $222 \mathrm{~nm}$ must in part reflect a lucky cancellation of errors, since the computed rotational distribution is slightly narrower than the experimental one and its primary maximum is a few quanta too high. The relative contributions of $\mathrm{A}$ and $\mathrm{B}$ absorptions must nonetheless be fairly accurate.

Suits and co-workers ${ }^{10}$ also measured $\beta$ at $288 \mathrm{~nm}$ in the far red wing of the OCS absorption, finding that vibrationally excited states made important contributions to the absorption and that $\beta$ was negative. We have not addressed these measurements in detail but they are consistent with the increasing contribution of the B state we find at very long wavelengths.

\section{Alignment parameter}

The alignment parameter $A_{0}^{(2)}$ or its corresponding bipolar moment $\beta_{0}^{2}(02)$ is a particularly incisive observable for determining the relative contributions of $A^{\prime}$ and $A^{\prime \prime}$ state absorptions to the photodissociation. While the anisotropy parameter $\beta$ must be -1 for $A^{\prime \prime}$ absorption, it can take values ranging from -1 to 2 for $A^{\prime}$ absorption depending on the transition dipole orientation and the post-excitation dynamics. Its interpretation is therefore somewhat clouded. The alignment parameter suffers no such fuzziness for a triatomic, $J=0$ classical model; it takes limiting negative values for $A^{\prime}$ absorption and limiting positive values for $A^{\prime \prime}$. The only data available are those of Sivakumar et $a .^{7}$ at $222 \mathrm{~nm}$, shown in the center right panel of Fig. 5 in the $\beta_{0}^{2}(02)$ form whose limits are $-1 / 2$ and +1 . The figure also shows as a solid black line their estimate of the fractional contribution of $A^{\prime \prime}$ absorption. The computed values agree with the measured ones remarkably well; again, there must be some fortuitous cancellation of errors in our computed results, but the agreement convinces us that our current treatment is qualitatively accurate.

\section{Dynamical analysis}

The upper panels of Fig. 5 show the orientation angles $\phi_{\mu}$ of the transition dipole moment for A state excitation and $\phi_{v}$ of the final $\mathrm{S}$ atom velocity for excitation to both $\mathrm{A}$ and B states. Both quantities are presented as weighted averages over all trajectories in each rotational bin. They are measured with respect to the initial $R$ vector as shown in Fig. 6. In this coordinate system direct axial recoil from a linear molecule would give $\phi_{v}=180^{\circ}$, and similarly a transition dipole moment along the axis of a linear molecule would give $\phi_{\mu}=180^{\circ}$.

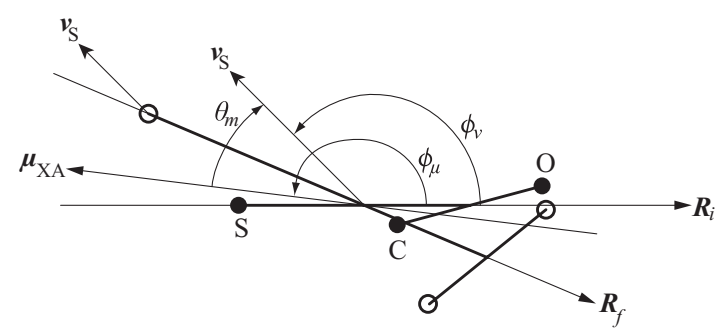

FIG. 6. Definitions of angles. The solid dots give the initial atomic positions, and the open circles indicate their positions after the forces have become negligible. Not to scale. 
The average transition dipole orientation for the A state is relatively flat from low $j$ up to the central minimum of the rotational distribution at each wavelength. At $248 \mathrm{~nm}$ the transition dipole is displaced about $25^{\circ}$ from the axis, and the displacement increases to $33^{\circ}, 39^{\circ}$, and $41^{\circ}$ at the shorter wavelengths. This behavior is consistent with the data in Table I; since the A state has a bent geometry, excitation at shorter wavelengths selects OCS molecules nearer linearity, and for these the transition dipole is oriented farther away from the axis. In the upper part of the rotational distribution, $\phi_{\mu}$ shows more sudden changes, presumably because only a subset of initial geometries can reach the avoided crossing region and make transitions to the $\mathrm{X}$ state.

The fragment velocity direction $\phi_{v}$ shows a relatively simple behavior with $j$. In the main part of the rotational distribution, it decreases slowly with increasing $j$, and the behavior is essentially the same on the A and B surfaces. This slow change arises from the increased torque felt by molecules dissociating to higher $j$, and its basic physics is captured by the empirical models used by Demyanenko et al.$^{46}$ and Brouard et $a l .{ }^{3}$ In the upper part of the rotational distribution, representing only excitation to the A state followed by transitions to $\mathrm{X}$, the deflection angle changes more steeply for the same reason.

In our calculations $\phi_{v} \approx \phi_{\mu}$ for $j \approx 42$ at $248 \mathrm{~nm}$ and for $j \approx 54$ at $235 \mathrm{~nm}$. One would expect $\beta$ to increase slowly with $j$ below those values, and decrease with $j$ above them, if the transition dipole direction was fixed and excitation occurred only to a single state. The A-state-specific values $\beta_{\mathrm{A}}$ shown in red in the center panels of Fig. 5 do show just that behavior. Since $\cos ^{2} \theta_{\mathrm{m}}$ does not vary very much for $0 \leq \theta_{\mathrm{m}} \lesssim 20^{\circ}, \beta$ remains near its limiting value across most of the rotational distribution for pure A-state excitation despite the modest variations in $\theta_{\mathrm{m}}=\phi_{v}-\phi_{\mu}$. Janssen and coworkers ${ }^{47}$ reached the same conclusion through analysis of their imaging experiments, stating "the deflection angle [our $\phi_{v}$ ] depends on the rotational state of the $\mathrm{CO}(j)$ photofragment only to a small extent." At the very highest $j$, where there are steeper changes in $\phi_{v}$, this general observation no longer holds and rapid changes in nonaxial recoil do produce rapid changes in $\beta$.

The high values of $\beta$ observed in the upper part of the rotational distribution, and throughout the pure A-state results, do not reflect axial recoil from a near-linear molecule excited via a transition dipole aligned with the molecular axis. Nonaxial recoil is substantial at every $j$ for every dissociation wavelength we have examined. Instead, the product velocity vector and the transition dipole moment are both displaced from the molecular axis but by similar amounts, so that $\beta$ remains high throughout the A-state rotational distribution. The main source of the rapid changes in $\beta$ in the central part of the rotational distribution is not variations in nonaxial recoil, but instead changes in the fractional contribution of the B-state absorption in different parts of the distribution, as originally proposed and quantified by Sivakumar et $\mathrm{al}^{7}$

Janssen and co-workers, ${ }^{47}$ using imaging of $\mathrm{CO}$ photofragments from $230 \mathrm{~nm}$ dissociation of oriented OCS in the $v_{2}=1,|J l M\rangle=|111\rangle$ state, concluded that the $j=62$ fragment arose from OCS molecules with an average angle of $31^{\circ}$ between the transition dipole and the permanent dipole of the molecule. The corresponding angle from our calculations for excitation from the OCS ground state, assuming that the average permanent dipole moment lies along $R_{i}$, is $37^{\circ}$. Janssen et al ${ }^{47}$ also concluded that (in our notation) $\phi_{v} \approx \phi_{\mu}-16^{\circ}$ at $j=62$. We find $\phi_{v}<\phi_{\mu}$ (that is, the outgoing fragment is displaced from the initial molecular axis by more than the transition dipole) for high $j$ at longer wavelengths, but $\phi_{v} \sim \phi_{\mu}$ for $j=62$ at $230 \mathrm{~nm}$. Our physical pictures of the dissociation are therefore in reasonable but not perfect agreement. These modest disagreements probably have several origins. The most important may be the initial OCS vibrational excitation in the experiment whose effects we have not accounted for. This $l=1$ bending excitation should have modest influences on the average orientation of the transition moment and on the observed $\beta$ parameters. (It could have a strong influence on alignment parameters, which Janssen et al..$^{47}$ did not measure, because it invalidates the assumption that the triatomic plane does not rotate during the dissociation). Differences between the direction of the permanent dipole and $R_{i}$ vectors, errors in our potential surfaces and our classical model, and errors in the approximations made during the experimental data interpretation may also contribute.

Brouard $\mathrm{et} \mathrm{al}^{3}$ reached a different conclusion about the origin of the variation in $\beta$ with $j$. They showed that a model that assumed excitation only to the A state could fit several sets of $\beta(j)$ data purely because of variations in nonaxial recoil. To fit the increase of $\beta$ with $j$ they chose transition dipole moment orientations that were far from the molecular axis so that as the nonaxial recoil increased with increasing $j$, the final product velocity vectors moved toward the transition dipole vector until the velocity and transition dipole vectors were nearly parallel at the high end of the rotational distribution. These choices led to orientation angles of the transition dipole moment substantially farther from the molecular axis than Janssen et al.$^{47}$ suggested or that we have found here. At 248, 230, and $220 \mathrm{~nm}$ they estimated transition dipole orientations corresponding to $\phi_{\mu}=108^{\circ}, 115^{\circ}$, and $100^{\circ}$ respectively. The quality of their fits was good, but in light of the information on the extent of nonaxial recoil provided by our trajectories, we now feel that the necessary transition dipole orientations were unrealistic. The A-state-only model also cannot be easily reconciled with the angular momentum alignment results of Sivakumar t $_{\text {al. }}{ }^{7}$ These flaws are, of course, shared by our own earlier claim ${ }^{19}$ that absorption to $A^{\prime \prime}$ states was unimportant.

\section{SUMMARY AND CONCLUSIONS}

This paper has reported two main changes to our earlier treatment of the OCS photodissociation. First, we modified the electronic structure procedure used for computation of transition dipole moments between the ground and the excited singlet states. The new procedure still uses the MRCI approach based on a CASSCF reference wavefunction, but obtains the CASSCF orbitals by optimizing the average energy of a larger number of electronic states. This change resulted in a substantially larger transition dipole moment to the ${ }^{1} A^{\prime \prime}$ (B) state, and also yielded different orientations of the 
TDM vector for the ${ }^{1} A^{\prime}$ (A) state. Second, we applied an empirical modification to the $\mathrm{A}$ and $\mathrm{B}$ state potential energy surfaces in the region far from linearity, increasing the strength of the repulsive force. The modification was chosen to bring the rotational state distribution computed for absorption to the A state, which had a single maximum at too high a $j$, into reasonable agreement with experiment at $235 \mathrm{~nm}$. Neither of these changes notably affected the absorption spectrum or the very cold product vibrational distribution, which had already agreed well with experiment.

With these modifications, our theoretical model agrees at least semiquantitatively with measured product state and angular distributions from 222 to $248 \mathrm{~nm}$, and also with the angular momentum alignment parameters measured at $222 \mathrm{~nm}$. Comparison to the anisotropy and alignment measurements was the key indicator of the flaws in our earlier transition dipole moment functions; the absorption spectrum and the rotational and vibrational distributions could all be treated satisfactorily with models that include only the A state and its nonadiabatic coupling to $\mathrm{X}$.

Absorption to the $\mathrm{B}$ state contributes roughly half the photodissociation cross section in the main section of the product state distribution, in agreement with the earlier analyses of Sivakumar et $a l^{7}$ and van den Brom et $a l^{47}$ and in contrast to earlier claims by us ${ }^{18,19}$ and others ${ }^{3}$ that it was unimportant. The second, high- $j$ maximum in the rotational distribution arises from initial excitation to the A state and subsequent nonadiabatic transitions to the ground state, as suggested by Suzuki et al. ${ }^{8}$ While the anisotropy parameters $\beta$ are large in this upper part of the rotational distribution, nonaxial recoil is important but is compensated by displacements of the transition dipole moment away from the molecular axis.

\section{ACKNOWLEDGMENTS}

G.C.M. is grateful for advice from Gregory Hall, Peter Rakitzis, and Jesse Lutz.

${ }^{1}$ C. Brühl, J. Lelieveld, P. J. Crutzen, and H. Tost, Atmos. Chem. Phys. 12, 1239 (2012)

${ }^{2}$ J. A. Schmidt, M. S. Johnson, S. Hattori, N. Yoshida, S. Nanbu, and R. Schinke, Atmos. Chem. Phys. 13, 1511 (2013).

${ }^{3}$ M. Brouard, A. V. Green, F. Quadrini, and C. Vallance, J. Chem. Phys. 127, 084304 (2007).

${ }^{4}$ M. L. Lipciuc, T. P. Rakitzis, W. L. Meerts, G. C. Groenenboom, and M. H. M. Janssen, Phys. Chem. Chem. Phys. 13, 8549 (2011).

${ }^{5}$ L. T. Molina, J. J. Lamb, and M. J. Molina, Geophys. Res. Lett. 8, 1008, doi:10.1029/GL008i009p01008 (1981).

${ }^{6}$ C. Y. R. Wu, F. Z. Chen, and D. L. Judge, J. Quant. Spectrosc. Radiat. Transf. 61, 265 (1999).

${ }^{7}$ N. Sivakumar, G. E. Hall, P. L. Houston, J. W. Hepburn, and I. Burak, J. Chem. Phys. 88, 3692 (1988).

${ }^{8}$ T. Suzuki, H. Katayanagi, S. Nanbu, and M. Aoyagi, J. Chem. Phys. 109, 5778 (1998)
${ }^{9}$ G. Nan, I. Burak, and P. L. Houston, Chem. Phys. Lett. 209, 383 (1993).

${ }^{10}$ M. H. Kim, W. Li, S. K. Lee, and A. G. Suits, Can. J. Chem. 82, 880 (2004).

${ }^{11}$ T. P. Rakitzis, A. J. van den Brom, and M. H. M. Janssen, Science 303, 1852 (2004).

${ }^{12}$ S. K. Lee, R. Silva, S. Thamanna, O. S. Vasyutinskii, and A. G. Suits, J. Chem. Phys. 125, 144318 (2006).

${ }^{13}$ R. Schinke, J. Chem. Phys. 134, 064313 (2011).

${ }^{14}$ J. A. Schmidt, M. S. Johnson, U. Lorenz, G. C. McBane, and R. Schinke, J. Chem. Phys. 135, 024311 (2011).

${ }^{15}$ J. A. Schmidt, M. S. Johnson, and R. Schinke, Atmos. Chem. Phys. 11, 8965 (2011).

${ }^{16}$ R. Schinke, J. A. Schmidt, and M. S. Johnson, J. Chem. Phys. 135, 194303 (2011).

${ }^{17}$ G. C. McBane and R. Schinke, J. Chem. Phys. 136, 044314 (2012).

${ }^{18}$ J. A. Schmidt, M. S. Johnson, G. C. McBane, and R. Schinke, J. Chem. Phys. 136, 131101 (2012).

${ }^{19}$ J. A. Schmidt, M. S. Johnson, G. C. McBane, and R. Schinke, J. Chem. Phys. 137, 054313 (2012).

${ }^{20}$ H.-J. Werner and P. J. Knowles, J. Chem. Phys. 89, 5803 (1988).

${ }^{21}$ P. J. Knowles and H.-J. Werner, Chem. Phys. Lett. 145, 514 (1988).

${ }^{22}$ S. Hattori, S. O. Danielache, M. S. Johnson, J. A. Schmidt, H. G. Kjaergaard, S. Toyoda, Y. Ueno, and N. Yoshida, Atmos. Chem. Phys. 11, 10293 (2011).

${ }^{23}$ H.-J. Werner and P. J. Knowles, J. Chem. Phys. 82, 5053 (1985).

${ }^{24}$ P. J. Knowles and H.-J. Werner, Chem. Phys. Lett. 115, 259 (1985).

${ }^{25}$ T. H. Dunning, Jr., J. Chem. Phys. 90, 1007 (1989).

${ }^{26}$ J. Thom H. Dunning, K. A. Peterson, and A. K. Wilson, J. Chem. Phys. 114, 9244 (2001).

${ }^{27}$ H.-J. Werner, P. J. Knowles, F. R. Manby, M. Schütz et al., MOLPRO, version 2010.1, a package of ab initio programs, 2010, see http://www.molpro.net.

${ }^{28}$ R. Schinke and J. A. Schmidt, J. Phys. Chem. A 116, 11083 (2012).

${ }^{29}$ T. Korona and H.-J. Werner, J. Chem. Phys. 118, 3006 (2003).

${ }^{30}$ J. A. Schmidt, "Transition dipoles with MRCI: On the importance of higher excited states" (unpublished).

${ }^{31}$ R. Schinke, Photodissociation Dynamics (Cambridge University Press, Cambridge, 1993).

${ }^{32}$ R. Schinke, J. Suarez, and S. C. Farantos, J. Chem. Phys. 133, 091103 (2010).

${ }^{33}$ S. Y. Grebenshchikov, Z.-W. Qu, H. Zhu, and R. Schinke, Phys. Chem. Chem. Phys. 9, 2044 (2007).

${ }^{34}$ J. C. Tully, J. Chem. Phys. 93, 1061 (1990).

${ }^{35}$ R. Schinke and G. C. McBane, J. Chem. Phys. 132, 044305 (2010).

${ }^{36}$ A. J. van den Brom, T. P. Rakitzis, J. van Heyst, T. N. Kitsopoulos, S. R. Jezowski, and M. H. M. Janssen, J. Chem. Phys. 117, 4255 (2002).

${ }^{37}$ T. P. Rakitzis, P. C. Samartzis, and T. N. Kitsopoulos, J. Chem. Phys. 111, 10415 (1999).

${ }^{38}$ Z. H. Kim, A. J. Alexander, and R. N. Zare, J. Phys. Chem. A 103, 10144 (1999).

${ }^{39}$ T. P. Rakitzis, P. C. Samartzis, and T. N. Kitsopoulos, Phys. Rev. Lett. 87, 123001 (2001)

${ }^{40}$ A. J. van den Brom, T. P. Rakitzis, and M. H. M. Janssen, J. Chem. Phys. 123, 164313 (2005).

${ }^{41}$ Y. Mo, H. Katayanagi, M. C. Heaven, and T. Suzuki, Phys. Rev. Lett. 77, 830 (1996).

${ }^{42}$ R. N. Zare, Angular Momentum (Wiley, New York, 1988).

${ }^{43}$ G. C. McBane, L. T. Nguyen, and R. Schinke, J. Chem. Phys. 133, 144312 (2010).

${ }^{44}$ R. N. Dixon, Acc. Chem. Res. 24, 16 (1991).

${ }^{45}$ R. N. Dixon, J. Chem. Phys. 85, 1866 (1986).

${ }^{46}$ A. V. Demyanenko, V. Dribinski, H. Reisler, H. Meyer, and C. X. W. Qian, J. Chem. Phys. 111, 7383 (1999).

${ }^{47}$ A. J. van den Brom, T. P. Rakitzis, and M. H. M. Janssen, J. Chem. Phys. 121, 11645 (2004). 